\title{
Vesicle-associated protein 1: a novel ovarian immunocontraceptive target in the common brushtail possum, Trichosurus vulpecula
}

\author{
A Nation, S Cui and L Selwood \\ Department of Zoology, The University of Melbourne, Parkville, 3010 Victoria, Australia \\ Correspondence should be addressed to A Nation; Email: a.nation@pgrad.unimelb.edu.au
}

\begin{abstract}
Ovarian-based immunological research is currently restricted to proteins of the zona pellucida. This study examined the immunocontraceptive potential of a novel vesicle-associated protein, VAP1, previously isolated from the vesicle-rich hemisphere of the brushtail possum oocyte. Seven female possums were immunized against recombinant glutathione $S$-transferase-VAP1 fusion protein. Control animals $(n=3)$ received antigen-free vaccinations. Following immunization, regular blood sampling determined the level and duration of immune response. Animals were monitored daily, pre- and post-immunization, to determine estrous cycling activity and the percentage of reproductive cycles yielding viable young. The reproductive tracts and somatic organs of VAP1-immunized ( $n=7)$, $\operatorname{control-}$ immunized $(n=3)$ and non-immunized $(n=5)$ animals were collected and examined by histology and transmission electron microscopy. VAP1 immunization caused a strong and sustained immune response. Elevated levels of VAP1 antibody binding were detected in sera following initial injections, and immune titers rose as boosters were administered. Immunization had no adverse effect upon animal behavior or body condition. Immunized females demonstrated no major change in annual estrous cycling activity; however, the percentage of reproductive cycles resulting in pouch young decreased significantly $(P<0.05)$ by $40 \%$. Histological and ultrastructural analyses revealed an abundance of lipid-like degradation bodies within the ooplasm of developing oocytes and the cytoplasm of failing uterine zygotes. Active macrophage invasion of enlarged endometrial glands was observed in the uteri of two females. Reproductive tract changes are discussed in relation to observed fertility decline. The results of this study indicate that VAP1 has exciting potential as an immunocontraceptive target for possum control in New Zealand.
\end{abstract}

Reproduction (2008) 136 657-665

\section{Introduction}

Manipulation of fertility, via immunocontraception, has been the subject of intense research for many years. Contraceptive vaccines may interfere with gamete production, fertilization, or embryogenesis by stimulating an immune reaction to key reproductive molecules. Common targets of interest include proteins of the zona pellucida (Barber \& Fayrer-Hosken 2000), sperm surface antigens, and reproductive hormones (Naz et al. 2005, Cooper \& Larsen 2006, Hardy \& Braid 2007).

The zona pellucida (ZP) plays a critical role in mammalian fertilization, hence ZP glycoproteins are the most frequently proposed candidates for ovarianbased immunocontraception (Barber \& Fayrer-Hosken 2000). Numerous trials have validated the contraceptive potential of various antigens (Frank et al. 2005); however, fertility reduction is frequently accompanied by ovarian pathology (Mahi- Brown et al. 1988, Curtis et al. 2007) and hormonal disturbance (Skinner et al.
1984, Stoops et al. 2005). An ideal ovarian immunological agent would inhibit fertility without causing ovarian dysfunction.

A major obstacle in developing contraceptive vaccines for the regulation of wildlife populations is the risk of inadvertently affecting non-target species. The situation in New Zealand is unusual in that the country's major mammalian pest is an introduced marsupial, the common brushtail possum (Trichosurus vulpecula). As New Zealand has no native marsupials, the development of a marsupial-specific contraceptive vaccine, derived from a unique feature of marsupial development, such as oocyte-conceptus polarity, would eliminate the threat to endemic species.

In marsupials, polarity is initially established in primary oocytes by the eccentric, peripheral location of the nucleus (Selwood 1992). Polarity is further enhanced in many species by the accumulation of conspicuous membrane-bound electron-lucent vesicles opposite the nucleus, either during oogenesis or following fertilization (Selwood 1994). Oocyte vesicles 
originate via the fusion of multivesicular bodies, derived from endoplasmic, endocytotic, and Golgi vesicles in Monodelphis domestica (Falconnier \& Kress 1992) as well as coated and heterogeneous vesicles in Isoodon macrourus (Ullmann \& Butcher 1996). Although the chemical composition of these vesicles remains largely unknown, there is some evidence they contain glycoproteins (Kress 1996) and saccharide residues (Breed 1996). Oocyte and zygote polarity is maintained by species-specific cytoskeletal architecture (Breed 1994, Merry et al. 1995, Frankenberg \& Selwood 1998).

During early cleavage, vesicular contents are generally shed in a polarized fashion into the perivitelline space (Breed \& Leigh 1990, Taggart et al. 1993, Sathananthan et al. 1997), contributing to blastocoel formation (Frankenberg \& Selwood 2001). Cell-zona attachment first occurs in the opposite hemisphere to vesicle discharge (Selwood 1992, 2001, Merry et al. 1995), where the pluriblast (future embryonic) cells develop. Trophoblast (future placental) cells line the opposite hemisphere and continue to secrete vesicular matter into the cleavage cavity. In a number of marsupials, T. vulpecula (Frankenberg \& Selwood 1998), Sminthopsis macroura, and Antechinus stuartii (Selwood \& Smith 1990), cytoplasmic vesicles are unevenly distributed in the first two cell lineages of the embryo, and the polarized discharge of extracellular material appears essential for normal development (Frankenberg \& Selwood 1998, Kress \& Selwood 2003, 2004).

Recently, a number of vesicle-associated molecules (VAMs) have been identified in the vesicle-rich hemisphere of the T. vulpecula oocyte (Selwood et al. 1999). VAMs are associated with oocyte polarity, cell lineage allocation, and are believed to be essential for normal oogenesis, cleavage, and blastocyst formation. Vesicleassociated protein 1 (VAP1), a previously characterized ovarian-specific protein, has been proposed as a potential immunocontraceptive target for possum control in New Zealand (Cui et al. 2005). This study was conducted to assess the ability of VAP1 immunization to elicit an immune response in T. vulpecula and to examine the effect of vaccination on female fertility.

\section{Results}

\section{Animal health}

Ten reproductively mature female $T$. vulpecula were treated in this study, conducted between 2003 and 2007. Seven animals were immunized with glutathione $S$-transferase (GST)-VAP1, and three received control vaccines. Animals were monitored daily and on the basis of body condition, weight, and behavior the treatments did not appear detrimental to animal health. Two experimental animals developed slight swellings at the primary injection sites; however, no animals developed lesions, a common, undesirable side effect of Freund's complete adjuvant (FCA; Broderson 1989).

\section{Estrous cycling activity and fertility}

To determine whether female fertility was affected by VAP1 immunization, estrous cycling activity, mating, and birth data were collected from the same cohort of animals $(n=7)$, pre- and post-VAP1 immunization. In general, most females cycled continuously between March and September. Following immunization, however, animals were more likely to continue cycling during the months of October and November, indicating that VAP1 immunization may prolong the breeding season in some females. Female fertility (the percentage of estrous cycles resulting in birth) showed a monthly decline following immunization, with later months of the breeding season (May to October-November) most affected (Fig. 1). Despite continuing to cycle during October-November, no immunized females produced young during this period. Following immunization, individual fertility levels dropped in six of the seven treated animals (Fig. 2), and mean female fertility ( \pm S.E.M.) declined significantly $(t=10.0, \mathrm{df}=6, P \leq 0.01)$. Changes in fertility were not significantly correlated with animal age or estrous cycle stage at immunization $(P>0.05)$.

\section{Immune response}

An immune response to VAP1 was detected in the serum of five of seven VAP1-immunized females (Fig. 3). Unfortunately, a lack of protein prevented analysis of serum antibody titers for the final two experimental animals. An immune response was not detected in serum collected from any of the control-immunized females $(n=3)$. Booster VAP1 injections, administered at intervals from the primary immunization, caused a rapid secondary response with marked elevation of serum

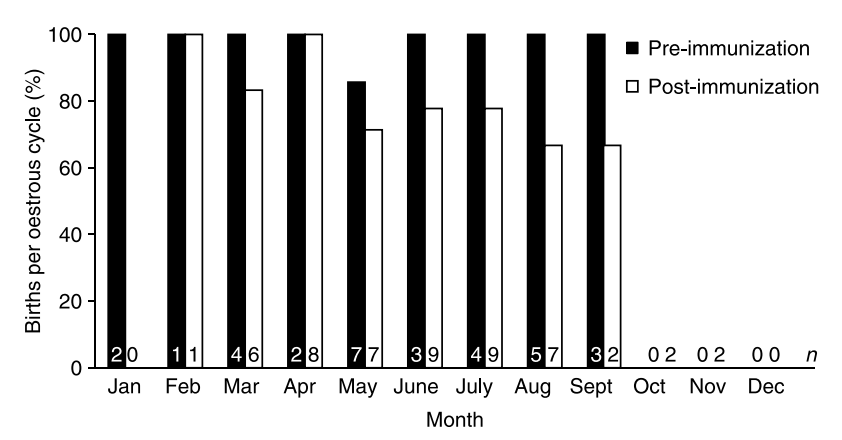

Figure 1 T. vulpecula annual estrous cycling activity before and after VAP1 immunization, collated from 2003 to 2007. The majority of females cycled continuously from March to September. The percentage of estrous cycles resulting in a birth declined after immunization, with later months of the breeding season (May to October-November) most affected. Despite continuing to cycle during October-November, no VAP1-immunized females produced young during this period. $n=$ Number of cycles recorded. 


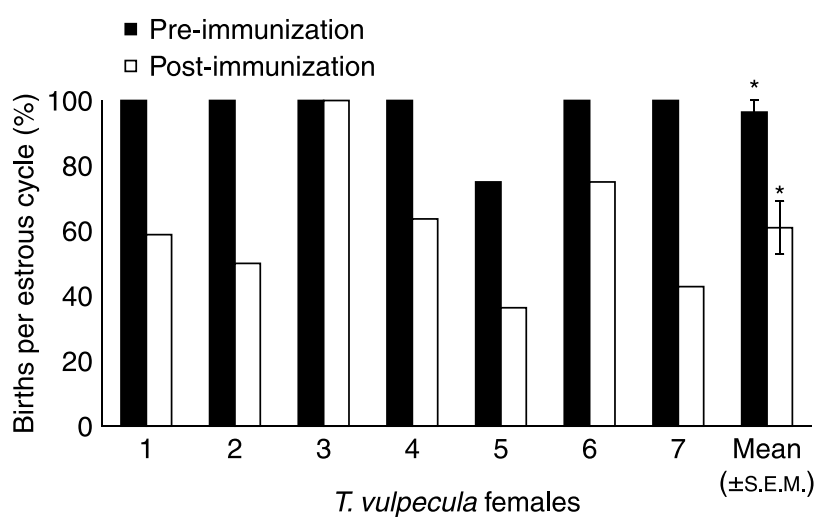

Figure 2 The percentage of estrous cycles resulting in a birth for individual T. vulpecula females (numbers 1-7), prior to and following VAP1 immunization. After immunization, fertility was reduced in six of the seven animals. Mean female fertility ( \pm s.E.M.) fell from $96 \%( \pm 3.6)$ pre-immunization to $56 \%( \pm 8.1)$ post-immunization, a reduction of $40 \%$. ${ }^{*}$ Significant difference $(t=10.0, \mathrm{df}=6, P \leq 0.01)$.

VAP1-antibody levels. The highest immune titers were detected 1-4 months after the initial immunization and varied between individuals. Additional booster injections, given to three females between 6 and 18 months from the primary immunization, caused a subsequent increase in immune response.

The percentage reduction in fertility per individual was positively correlated with the maximum immune titer reached $(R=0.769, n=5, P=0.037)$, indicating that the level of immune response determined the degree to which fertility was reduced (Fig. 4).

\section{Tissue response}

Histological examination of the lymphatic system and somatic tissue (heart, kidney, liver, lung, spleen, lymph nodes, and oviduct) of VAP1-immunized animals $(n=7)$

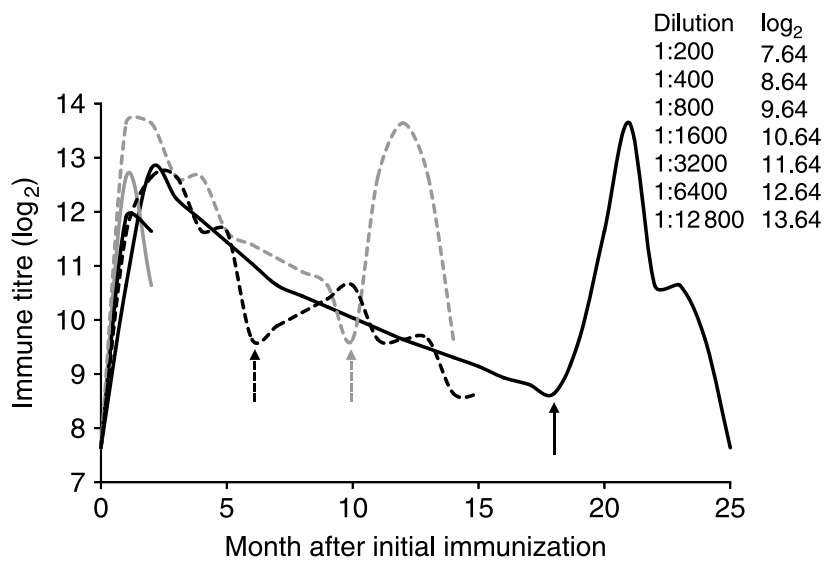

Figure 3 The immune titer $\left(\log _{2}\right)$ of five VAP1-immunized T. vulpecula females. Primary immunizations (Month 0 ) were followed by two booster injections (Months 1 and 2). The highest immune titers were recorded between 1 and 4 months from the initial immunization. Three females received an additional booster as indicated by the arrows. The final data point on each line indicates euthanasia of the animal.

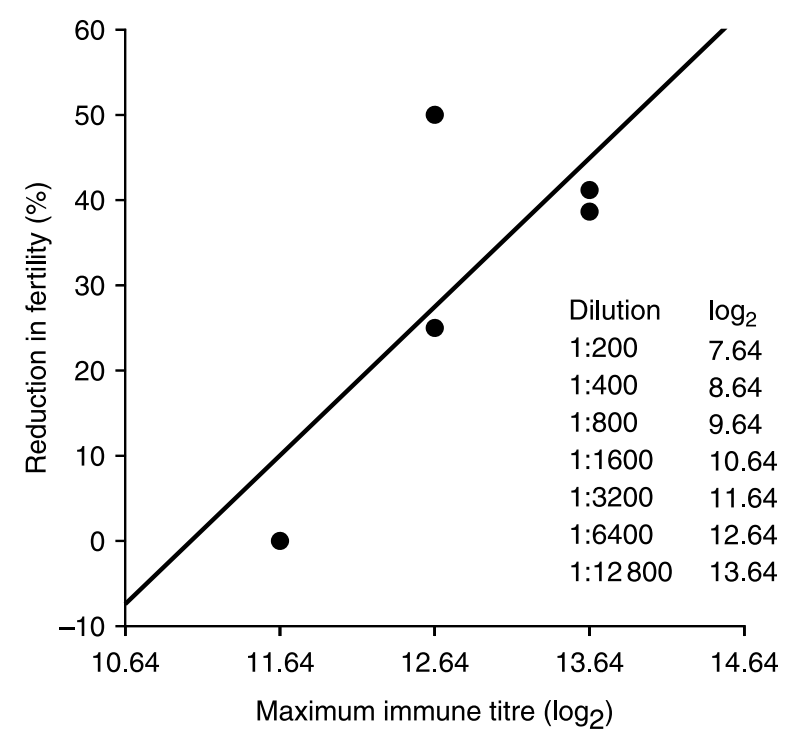

Figure 4 Following VAP1 immunization, the percentage reduction in fertility per $T$. vulpecula individual was positively correlated with the maximum immune titer reached $(R=0.769, n=5, P=0.037)$.

revealed no abnormalities when compared with tissue from control-immunized $(n=3)$ or non-immunized $(n=5)$ females. Normal uterine morphology, typical of early gestation, was observed in five of the seven VAP1immunized females (examined 0-8 days from ovulation). The uteri of the remaining two females (examined 7 days after removal of pouch young (RPY) and during anoestrus) showed greatly enlarged endometrial glands projecting into the uterine lumen (Fig. 5A). Large clusters of actively phagocytotic macrophages were present within the glandular lumen (Fig. 5B). Fertility reduction was greater than $40 \%$ in both these animals. All control-immunized $(n=3)$ and nonimmunized $(n=5)$ animals demonstrated normal uterine morphology with endometrial gland development typical of the reproductive stage examined.

In contrast to the ovaries of control-immunized $(n=3)$ and non-immunized $(n=5)$ animals, an abundance of oocyte inclusions, termed degradation bodies, were observed in the ovaries of four of the seven VAP1immunized females. Transmission electron micrography (TEM) revealed that the degradation bodies were lipid like in that they were bordered by an osmiophilic ring rather than a true, limiting trilaminar membrane. They contained defined electron-dense and electron-lucent regions, which presumably correlate with the saturated and unsaturated fatty acid content of the triglyceride and the degree of unsaturation of fatty acids present (Mehta \& Ghadially 1973). The degradation bodies were roughly spherical in shape, showed evidence of fusion with cytoplasmic vesicles, and were generally accompanied by regions of amorphous, electron-dense cytoplasm. T. vulpecula oocytes typically contain a few cytoplasmic lipid droplets of homogeneous appearance (Frankenberg 


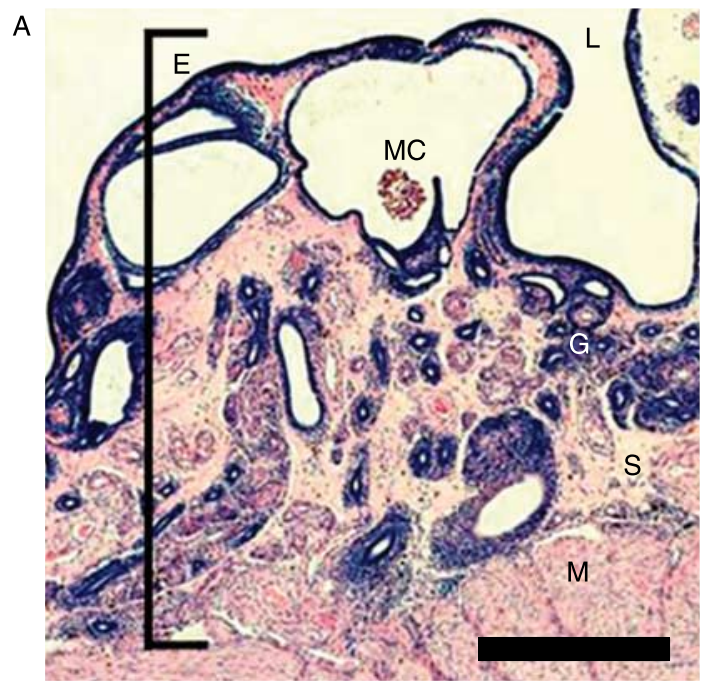

B

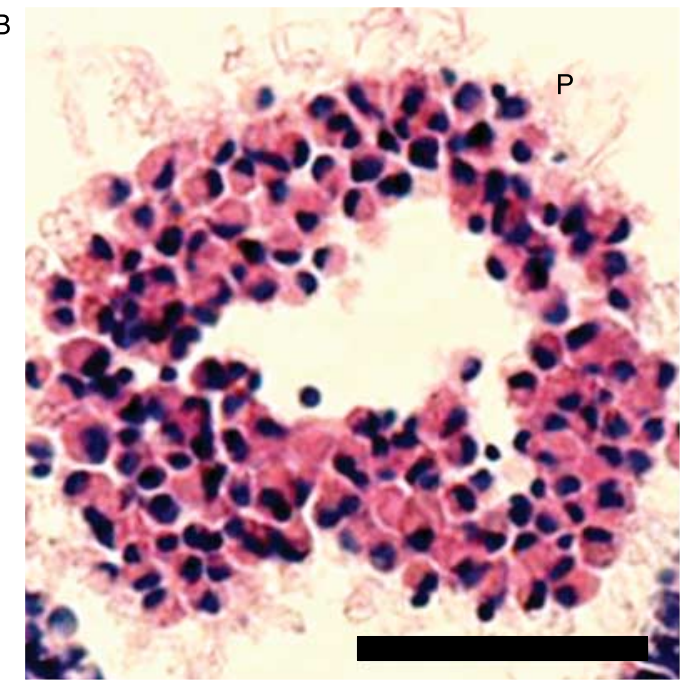

Figure 5 Light micrographs of uterine tissue from a VAP1-immunized T. vulpecula female $(8 \mu \mathrm{m}, \mathrm{H} \& \mathrm{E})$. (A) Transverse section through the uterus showing the uterine lumen $(\mathrm{L})$, glandular endometrium $(\mathrm{E})$, and muscular myometrium (M). Within the endometrial layer, several enlarged glands are visible and a cluster of macrophages (MC) can be seen in the glandular lumen. Normal glands (G), stromal tissue (S). Scale $=300 \mu \mathrm{m}$. (B) Higher magnification of clustered macrophages with pseudopodia $(\mathrm{P})$ within the lumen of an enlarged endometrial gland. Scale $=50 \mu \mathrm{m}$.

\& Selwood 2001). Normal lipid droplets were observed in the oocytes of all animals examined; however, degradation bodies (determined by the characteristics described above) were only observed in the oocytes of VAP1-immunized animals. Gross deposits of lipid of this nature imply fatty degeneration.

Degradation bodies first became apparent in primary oocytes after the transition of primordial to the primary follicle stage (Fig. 6A and B). As follicles developed, the inclusions increased in size and accumulated to various extents within the ooplasm (Fig. 6C-F). Analysis of primary oocyte cross-sections (from VAP1-immunized
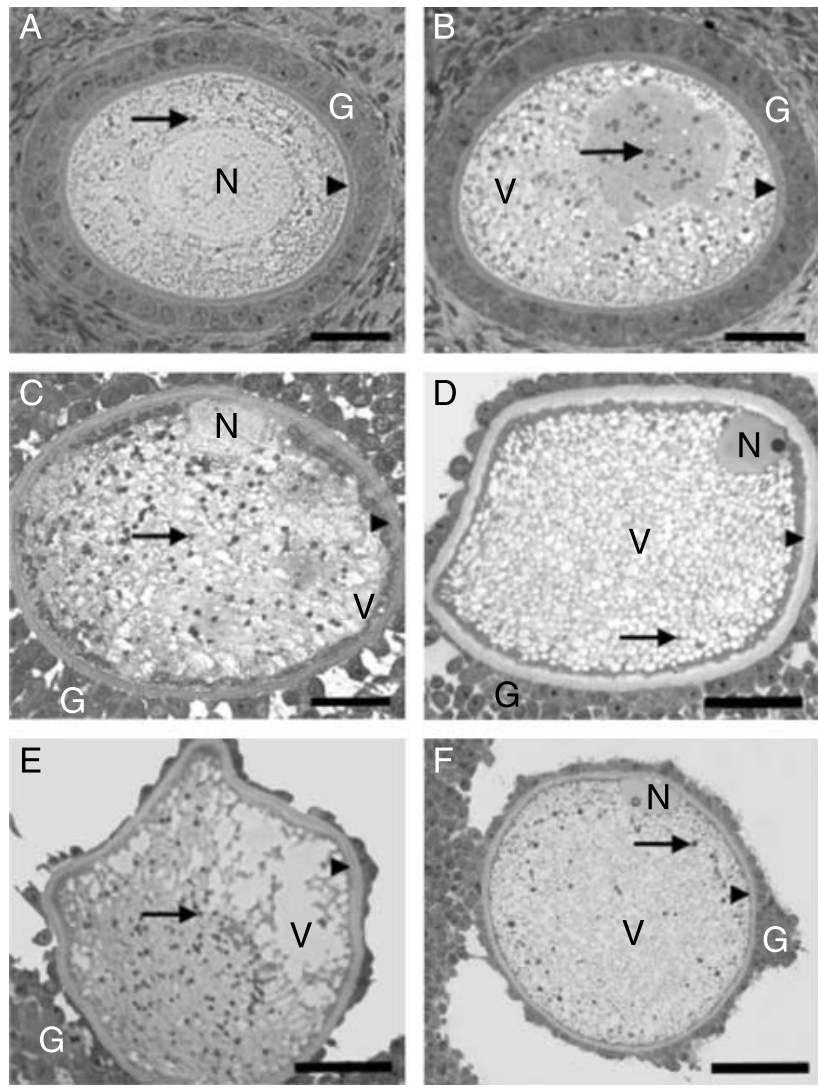

Figure 6 Light micrographs of oocytes from VAP1 immunized T. vulpecula females illustrating varying degrees of cytoplasmic lipid accumulation (normal lipid droplets and degradation bodies) during oogenesis ( $1 \mu \mathrm{m}$, Toluidine blue). (A) A normal primary oocyte (primary follicle) showing few cytoplasmic lipid droplets (arrow). Granulosa cells $(\mathrm{G})$, zona pellucida (arrow head), nucleus/germinal vesicle $(\mathrm{N})$. Scale $=15 \mu \mathrm{m}$. (B) An affected primary oocyte (primary follicle) showing elevated cytoplasmic lipid levels. Degradation bodies (arrow) are present throughout the oocyte. The largest degradation bodies are located in an area of amorphous, electrondense cytoplasm that appears almost devoid of vesicles (V).

Granulosa cells (G), zona pellucida (arrow head). Scale $=15 \mu \mathrm{m}$. (C) A badly affected primary oocyte (tertiary follicle) containing numerous degradation bodies (arrow), patches of amorphous, electron-dense cytoplasm, and an abnormally low level of vesicles (V). Granulosa cells $(\mathrm{G})$, zona pellucida (arrow head), nucleus/ germinal vesicle $(\mathrm{N})$. Scale $=15 \mu \mathrm{m}$. (D) A normal primary oocyte (early antral follicle) containing no degradation bodies, very few lipid droplets (arrow), an abundance of vesicles (V), and no areas of amorphous, electron-dense cytoplasm. Granulosa cells $(\mathrm{G})$, zona pellucida (arrow head), nucleus/germinal vesicle $(\mathrm{N})$. Scale $=15 \mu \mathrm{m}$. (E) A badly affected primary oocyte (antral follicle) in which the cytoplasm has segregated into two distinct areas, one containing degradation bodies (arrow) and amorphous, electron-dense cytoplasm, and the other coalesced vesicles (V). Granulosa cells (G), zona pellucida (arrow head). Scale $=26 \mu \mathrm{m}$. (F) A mildly affected antral follicle oocyte containing an abundance of vesicles interspersed with lipid and degradation bodies (arrow). No areas of amorphous, electron-dense cytoplasm are evident. Granulosa cells (G), zona pellucida (arrow head), nucleus/germinal vesicle $(\mathrm{N})$. Scale $=30 \mu \mathrm{m}$. 
and non-immunized females) revealed that the number of lipid inclusions (degradation bodies and normal lipid droplets) per $\mu \mathrm{m}^{2}$ oocyte cytoplasm was significantly greater in primary $(t=3.37, \mathrm{df}=44, P=0.02)$ and antral $(t=4.28, \mathrm{df}=15, P=0.02)$ follicle oocytes of VAP1immunized animals. The number of vesicles (greater or equal in size to the largest observed lipid inclusion) per $\mu \mathrm{m}^{2}$ oocyte cytoplasm was significantly reduced in primary follicle oocytes $(t=4.45, \mathrm{df}=44, P=0.12)$ of VAP1-immunized females but not in antral follicle oocytes $(P<0.05)$. Degradation bodies remained prominent in secondary oocytes and appeared largest in the most mature oocytes.

An unfertilized oocyte, two zygotes, and a late bilaminar blastocyst were obtained from the uteri of VAP1-immunized females. Both zygotes showed an intact, well-developed shell coat, with numerous spermatozoa embedded in the mucoid layer (Fig. 7A). The embryonic hemisphere was closely adhered to the ZP, whereas cytoplasm in the opposite, ab-embryonic hemisphere had become detached in places. Although cytoplasmic polarization was pronounced and yolk extrusion had begun, cytoplasmic fragmentation was evident and neither zygote appeared viable. In addition to normal cytoplasmic contents, such as filamentous, elongated mitochondria, electron-lucent vesicles and homogeneous, saturated lipid droplets, both zygotes contained a profusion of degradation bodies (Fig. 7B). Under TEM, the degradation bodies (Fig. 7C and D) appeared equivalent to those observed in the oocytes of VAP1-immunized animals.

\section{Discussion}

The immunization of $T$. vulpecula with GST-VAP1 elicited an oocyte-specific systemic antibody response and a cell-mediated uterine response, both novel reactions to an ovarian immunocontraceptive. In addition to a significant reduction in female fertility, VAP1 immunization caused embryonic failure and generated oocyte and uterine changes, suggesting that multiple mechanisms may contribute to fertility decline.

VAP1 is encoded by a unique gene with no significant homologies (Cui et al. 2005). Some regions of the protein, however, show structural similarity to the active regions of cystatin C, an endogenous cysteine proteinase inhibitor (Barrett et al. 1984, Turk \& Bode 1991, Dieckmann et al. 1993). Cystatin C, a member of the type 2 cystatin superfamily (Abrahamson et al. 2003), regulates cysteine proteinase activity in intra- and extracellular biological reactions (Barrett 1981). In addition to similar spatial form, the conserved presence of all five cystatin $C$ functional motifs (disulfide-paired cysteine residues) within the VAP1 sequence implies a homologous role. If so, VAP1 may contribute to extracellular matrix (ECM) stability during oogenesis and early development by allowing the accumulation
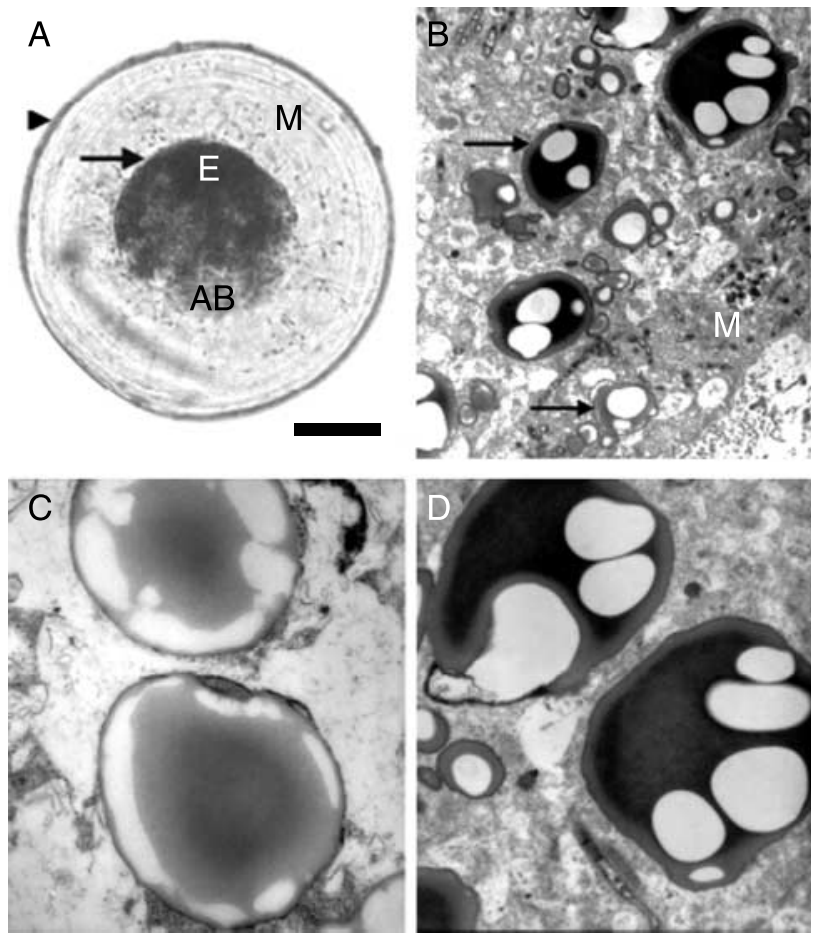

Figure 7 Light and transmission electron micrographs of a zygote retrieved from the active uterus of a VAP1 immunized $T$. vulpecula female. (A) The shell coat (arrow head) surrounding the zygote appears well-developed and numerous spermatozoa are embedded in the mucoid layer $(\mathrm{M})$. The embryonic hemisphere (E) of the zygote is closely adhered to the zona pellucida (arrow), whereas ooplasm in the opposite, ab-embryonic (AB) hemisphere is detached in places. Polar bodies (not shown) were located in the ab-embryonic hemisphere.

No cleavage furrow was observed. Scale $=100 \mu \mathrm{m}$. (B) A failed zygote fragment containing filamentous mitochondria $(M)$ and numerous degradation bodies (arrow) of varying size. $(\times 2200)$. (C) Smaller degradation bodies generally contained an electron-dense centre. Irregularly shaped, electron-lucent regions were located toward the periphery and bordered by a thin, osmiophilic ring. Evidence of fusion with cytoplasmic vesicles was regularly observed. ( $\times 21$ 000). (D) Larger degradation bodies contained central electron-lucent regions, contrasted with greater electron density in the surrounds $(\times 11500)$.

and stable storage of proteins within the oocyte cytoplasm, and/or stabilizing proteins as they are secreted into the blastocoel during cleavage (Cui et al. 2005).

Sparsely distributed lipid droplets have been described in the ooplasm of T. vulpecula (Frankenberg \& Selwood 2001), I. macrourus, I. obesulus, and Perameles nasuta (Ullmann 1981, Lyne \& Hollis 1983) and various eutherian species (Fleming \& Saacke 1972, Tesoriero 1981, Homa et al. 1986, Kikuchi et al. 2002). Although rare, degradation bodies have been observed in T. vulpecula zygote cytoplasm (Frankenberg \& Selwood 1998) but not during normal oogenesis (Frankenberg \& Selwood 2001). Degradation bodies of the size and magnitude observed here are previously unreported.

Despite the unusual oocyte changes induced by VAP1 immunization, local inflammatory cell levels were not 
elevated, therefore the ovarian immune response appears systemic. In contrast to ZP-based immunocontraceptives (Kitchener et al. 2002), VAP1 immunization affected only the germ cells of the ovary. The preservation of surrounding somatic tissue and normal ovarian morphology strengthens previous speculation that Vap1 expression may be oocyte specific (Cui et al. 2005). Following immunization, degradation bodies first became apparent in primary oocytes after the transition of primordial follicles to the primary follicle stage, correlating with a period of high Vap1 expression (Cui et al. 2005), and the initiation of oocyte vesicle formation (Frankenberg et al. 1996, Frankenberg \& Selwood 2001). The accumulation of degradation bodies in affected oocytes was associated with decreased vesicle number, suggesting that immunization compromises normal vesicular accumulation during oogenesis.

If VAP1 plays a role akin to cystatin C, then the transfer of VAP1 antibodies into the developing oocyte via serum would disrupt the normal cysteine proteinase-inhibitor balance, effectively allowing uncontrolled proteolytic activity of this type within the oocyte. In humans, imbalances between cathepsins of the cysteine proteinase family and their endogenous inhibitors are related to a variety of pathological conditions (Lenarcic et al. 1988, Henskens et al. 1996, Cimerman et al. 2001, Kos et al. 2001). We propose that the degradation bodies observed in this study are a direct result of increased proteolysis of oocyte storage proteins and/or ECM molecules due to VAP1 inhibition. During early development in T. vulpecula, the polarized discharge of ECM material appears important for embryonicabembryonic patterning (Frankenberg \& Selwood 1998, Kress \& Selwood 2003, 2004) and vesicles are preferentially distributed in the first two cell lineages (Frankenberg \& Selwood 1998). Decreased ECM stability, change in vesicular secretion and disruption of cell lineage allocation may all contribute to reducing embryonic success following immunization.

In addition to the antibody-mediated ovarian response, a small number of VAP1-immunized females demonstrated uterine changes consistent with endometrial cystic hyperplasia (De Bosschere et al. 2001), raising the possibility that a uterine immune response may contribute to this condition. The presence of macrophage clusters within enlarged endometrial glands suggests a cell-mediated reaction, possibly in response to the presence of a failed conceptus. All conceptuses obtained from the uteri of VAP1-immunized females showed cytoplasmic fragmentation; therefore, it appears probable that the macrophages observed were attacking the remains of a disintegrated oocyte or conceptus. There was no evidence to suggest that macrophages attack the intact conceptus, implying that the shell coat may have immunoprotectant properties. The absence of T lymphocytes within VAP1-immunized uteri is not unusual as experiments were generally scheduled during early pregnancy (prior to shell coat loss) in the hope of obtaining a conceptus. Due to the limited number of animals available, we were unable to examine all stages of pregnancy.

In conclusion, VAP1 immunization induces a novel oocyte-specific systemic immune response, which decreases female fertility without detrimental ovarian pathology. A secondary cell-mediated uterine response may also contribute to embryonic failure. Passive immune transfer (Adamski \& Demmer 2000), from immunized mothers to female young during lactation, may impart a generational effect. The ovary has a finite reserve of oocytes. As VAP1 targets all stages of oogenesis, immunization has the potential to impair fertility throughout an animal's lifespan. With the marsupialspecific VAP1 protein identified and shown to have an antibody mediated response to oocytes in vivo, VAP1 antibodies can now be used to carry fertility control agents into developing oocytes of the marsupial ovary.

\section{Materials and Methods}

\section{Animals}

Female T. vulpecula used in this study were housed in an outdoor colony maintained by L Selwood at The University of Melbourne. Colony maintenance and experiments followed Australian National Health and Medical Research Council Guidelines for the Care and Use of Animals for Scientific Purposes. A female-biased sex ratio $(\sim 2: 1)$ was preserved throughout the experimental period, and the colony contained an average of 18 individuals at any given time.

\section{Reproductive monitoring}

Adult females were monitored from March to November to establish the reproductive status. Estrous cycle stage was determined by cytology and mating success by the presence of spermatozoa in daily urine samples (Duckworth et al. 1999). T. vulpecula are monovular, polyoestrous seasonal breeders (Pilton \& Sharman 1962). Following RPY females return to estrus within 7-15 days (Duckworth et al. 1998). Estrus occurs 1-3 days prior to ovulation (Shorey \& Hughes 1973), and young are born 17.3 ( \pm 0.2 ) days post-coitus (Duckworth et al. 1998). Experimental females were monitored for 6-18 months before VAP1 immunization. Data obtained during this period served as respective (pre-immunized) controls for each individual, enabling the comparison of pre- and post-immunization activity. Immunizations were undertaken during the breeding season and post-immunization data were collected during the remainder of that season and the subsequent breeding season. The length of time animals were monitored post-immunization varied between individuals.

\section{Immunization}

Animals were randomly assigned to VAP1-immunized $(n=7)$ or control-immunized $(n=3)$ groups. The immunization 
antigen, recombinant GST-VAP1 fusion protein, was prepared according to a previous paper (Cui et al. 2005). The immunization protocol comprised an initial dose of $300 \mu \mathrm{g}$ GST-VAP1 in $500 \mu \mathrm{l}$ magnesium- and calcium-free PBS $^{-}$ emulsified with $500 \mu \mathrm{l}$ Freund's Complete Adjuvant (SigmaAldrich). Two booster vaccines followed at 28-day intervals, each containing $300 \mu \mathrm{g}$ GST-VAP1 in $500 \mu \mathrm{PBS}^{-}$in the presence of $500 \mu \mathrm{l}$ Freund's Incomplete Adjuvant (SigmaAldrich). Four animals received a further booster between 6 and 18 months from the initial immunization. Controlimmunized animals were treated over the same period with equivalent volumes of antigen-free $\mathrm{PBS}^{-}(n=2)$ or GST in PBS $^{-}(n=1)$ using the same adjuvants. Immunizations were delivered to multiple sites by s.c. injection. Prior to immunization and/or blood collection animals were sedated via i.m. injection with $10 \mathrm{mg} / \mathrm{kg}$ Zoletil (Virbac Animal Health, Peakhurst, NSW, Australia). Blood samples were collected from the lateral tail vein or the basilic forearm vein, prior to the initial injection (pre-immune control), 7-10 days after booster injections (Cooper \& Paterson 2008), and at 3 monthly intervals throughout the remaining experimental period. Prior to autopsy, animals were anesthetized with $15 \mathrm{mg} / \mathrm{kg}$ Zoletil delivered intramuscularly. The blood was collected via heart puncture and animals euthanized by intracardial injection of $150 \mathrm{mg} / \mathrm{kg}$ sodium pentobarbitone (Lethabarb; Virbac Animal Health). The blood samples were centrifuged at $2130 \mathrm{~g}$ for $10 \mathrm{~min}$. Sera were removed and stored at $-20{ }^{\circ} \mathrm{C}$ for analysis.

\section{ELISA}

The titer of polyclonal antibodies (against GST-VAP1) in the sera of VAP1-immunized $(n=7)$ and control-immunized $(n=3)$ animals was determined by ELISA, with pre-immune samples serving as negative controls for each individual. Assays were performed in 96-well ELISA plates (Immunlon $4 \mathrm{HBX}$; Thermo Scientific, Noble Park, VIC, Australia) using recombinant GSTVAP1 as the antigen. Non-specific binding was blocked with $3 \%$ albumin bovine serum (Sigma-Aldrich) and plates were washed between incubations with a buffering solution of 0.05\% Tween-20 (Selby Scientific, Notting Hill, VIC, Australia) in $\mathrm{PBS}^{-}$. Serial dilutions of the primary antibody, T. vulpecularaised polyclonal antisera, were detected by application of the secondary antibody, horseradish peroxidase (HRP)-conjugated protein A (GE Health Care, Castle Hill, NSW, Australia), diluted 1:5000 in $\mathrm{PBS}^{-}$. Incubation with tetramethyl benzidine substrate (Sigma-Aldrich), which reacts with HRP, produced an insoluble compound. Reactions were terminated after $30 \mathrm{~min}$ by the addition of $0.5 \mathrm{M}$ sulfuric acid. Color absorbance was measured at $450 \mathrm{~nm}$ using an ELISA reader (Multiscan EX; Thermo Electron Corporation, Vantaa, Finland). The three negative controls included on all plates comprised no primary antibody incubation, no secondary antibody incubation, and no primary or secondary antibody incubation.

Endpoint antibody titers were obtained from the lowest dilution factor at which the absorbance of post-immunization sera remained greater than the absorbance of pre-immunization sera (1:200 dilution). For analysis, endpoint dilution factors were expressed as $\log _{2}$ values (Doolin et al. 2002).

\section{Histology}

Tissue segments (heart, kidney, liver, lung, spleen, lymph node, ovary, oviduct, and uterus) were collected from all immunized animals and fixed by immersion in $4 \%$ paraformaldehyde (PFA; Merck) in PBS $^{-}$for $24 \mathrm{~h}$, dehydrated in ethanol, embedded in paraffin, serially sectioned at $6-8 \mu \mathrm{m}$, mounted, and stained with Harris' Hematoxylin and Putt's Eosin. Tissue morphology was compared between VAP1-immunized $(n=7)$ and controlimmunized $(n=3)$ animals. The tissue from healthy, nonimmunized colony animals $(n=5)$ was also collected, processed in the same manner, and used as a reference for normal morphology.

\section{Transmission electron microscopy}

Ovarian follicles, oocytes, and conceptuses were immersed in Superfix $(2.5 \%$ glutaraldehyde (ProSciTech, Thuringowa, QLD, Australia), 3\% PFA, 0.2 M sodium cacodylate buffer) at RT for $2 \mathrm{~h}(\mathrm{pH} 7.4)$. After three rinses in $0.1 \mathrm{M}$ sodium cacodylate buffer $(\mathrm{pH} 7.4)$, post-fixation took place in $1 \%$ osmium tetroxide (ProSciTech) diluted with $0.2 \mathrm{M}$ sodium cacodylate buffer for $2 \mathrm{~h}$ at RT. Following rinsing in $0.1 \mathrm{M}$ sodium cacodylate buffer, the tissue was dehydrated in an ethanol series, infiltrated with a propylene oxide-Epon araldite mixture, embedded in pure Epon araldite, and polymerized at $60{ }^{\circ} \mathrm{C}$ for $48 \mathrm{~h}$. The resin consisted of a mixture of $25 \mathrm{ml}$ Procure 812, $15 \mathrm{ml}$ Araldite 502, $55 \mathrm{ml}$ dodecenyl succinic anhydride, and $1.25 \mathrm{ml}$ benzyldimethylamine (ProSciTech).

Semi-thin sections $(1 \mu \mathrm{m})$ were cut with glass knives on an ultramicrotome (Ultracut E; Reichert-Jung, Heidelberg, Germany) and stained with $1 \%$ toluidine blue and $1 \%$ sodium borate in distilled water at $90{ }^{\circ} \mathrm{C}$ for $1-2 \mathrm{~min}$. At least three resin-embedded ovarian segments were serially sectioned, for each VAP1-immunized $(n=7)$ and non-immunized $(n=5)$ animal. Primary oocyte diameters, in primary and antral follicles, were measured at their largest cross-section, and counts made of the number of lipid inclusions (degradation bodies and normal lipid droplets) and the number of vesicles (greater or equal in size to the largest lipid inclusion) within the oocyte cytoplasm.

Thin sections $(70 \mathrm{~nm})$, cut with a diamond knife, were contrasted with $3 \%$ uranyl acetate and $0.6 \%$ lead citrate, mounted on coated grids and examined with a transmission electron microscope (CM 10; Philips, Eindhoven, The Netherlands).

\section{Statistical analysis}

Paired-samples $t$-tests were used to compare female fertility (i.e., the percentage of estrous cycles resulting in birth), preand post-VAP1 immunization. The correlation between individual fertility reduction and maximum immune titer reached was determined by linear regression. Independentsamples $t$-tests compared the number of lipid inclusions and the number of vesicles per $\mu \mathrm{m}^{2}$ oocyte cytoplasm between VAP1-immunized and non-immunized animals. 


\section{Declaration of interest}

We declare that there is no conflict of interest that could be perceived as prejudicing the impartiality of the research.

\section{Funding}

This work was supported by a grant from the Foundation for Research Science and Technology, New Zealand (MELB0301), and The University of Melbourne, Australia.

\section{Acknowledgements}

Many thanks are due to Kamani Nanayakkara and Ellen Menkhorst for assistance with animal work, and Joan Clark for assistance with electron micrography.

\section{References}

Abrahamson M, Alvarez-Fernandez M \& Nathanson CM 2003 Cystatins. Proteases and the Regulation of Biological Processes 70 179-199.

Adamski F \& Demmer J 2000 Immunological protection of the vulnerable marsupial pouch young: two periods of immune transfer during lactation in Trichosurus vulpecula (brushtail possum). Developmental and Comparative Immunology 24 491-502.

Barber M \& Fayrer-Hosken R 2000 Possible mechanisms of mammalian immunocontraception. Journal of Reproductive Immunology $\mathbf{4 6}$ 103-124.

Barrett A 1981 Cystatin, the egg-white inhibitor of cysteine proteinases. Methods in Enzymology 80 771-778.

Barrett A, Davies M \& Grubb A 1984 The place of human gamma-trace (cystatin-C) amongst the cysteine proteinase-inhibitors. Biochemical and Biophysical Research Communications 120 631-636.

De Bosschere H, Ducatelle R, Vermeirsch $H$, Van Den Broeck W \& Coryn M 2001 Cystic endometrial hyperplasia-pyometra complex in the bitch: should the two entities be disconnected? Theriogenology $\mathbf{5 5}$ 1509-1519.

Breed WG 1994 How does sperm meet egg? - in a marsupial. Reproduction, Fertility, and Development 6 485-506.

Breed WG 1996 Egg maturation and fertilization in marsupials. Reproduction, Fertility, and Development 8 617-643.

Breed WG \& Leigh CM 1990 Morphological changes in the oocyte and its surrounding vestments during in vivo fertilization in the dasyurid marsupial Sminthopsis crassicaudata. Journal of Morphology 204 177-196.

Broderson J 1989 A retrospective review of lesions associated with the use of Freund's adjuvant. Laboratory Animal Science 39 400-405.

Cimerman N, Mesko Brguljan P, Krasovec M, Suskovic S \& Kos J 2001 Serum concentration and circadian profiles of cathepsins $\mathrm{B}, \mathrm{H}$ and $\mathrm{L}$, and their inhibitors, stefins $\mathrm{A}$ and $\mathrm{B}$, in asthma. Clinica Chimica Acta; International Journal of Clinical Chemistry 310 113-122.

Cooper DW \& Larsen E 2006 Immunocontraception of mammalian wildlife: ecological and immunogenetic issues. Reproduction 132 821-828.

Cooper H \& Paterson Y 2008 Production of polyclonal antisera. Current Protocols in Immunology Chapter 2 11.12.1-11.12.10.

Cui S, Nikolovski S, Nanayakkara K \& Selwood L 2005 VAP1, with cystatin $\mathrm{C}$ motif, an oocyte protein encoded by a novel ovarian-specific gene during oogenesis in the common brushtail possum (Trichosurus vulpecula). Molecular Reproduction and Development 71 19-28.

Curtis P, Richmond M, Miller L \& Quimby F 2007 Pathophysiology of whitetailed deer vaccinated with porcine zona pellucida immunocontraceptive. Vaccine 25 4623-4630.

Dieckmann T, Mitschang L, Hofmann M, Kos J, Turk V, Auerswald E, Jaenicke R \& Oschkinat H 1993 The structures of native phosphorylated chicken cystatin and of a recombinant unphosphorylated variant in solution. Journal of Molecular Biology 234 1048-1059.
Doolin E, Wedlock D, Buddle B \& Skinner M 2002 Mucosal immunity in the brushtail possum (Trichosurus vulpecula): detection of antibody in serum and at female reproductive sites after intranasal immunization. Immunology and Cell Biology 80 358-363.

Duckworth J, Buddle B \& Scobie S 1998 Fertility of brushtail possums (Trichosurus vulpecula) immunised against sperm. Journal of Reproductive Immunology 37 125-138.

Duckworth J, Harris M, Mate K, McCartney C, Buist J, Scobie S, Jones D \& Jones S 1999 Development of brushtail possum immunocontraception: targeting sperm and egg antigens. In Advances in the Biological Control of Possums, pp 72-76. Ed. G Sutherland. Wellington, New Zealand: The Royal Society of New Zealand.

Falconnier C \& Kress A 1992 Ultrastructural aspects of oocyte growth in the marsupial Monodelphis domestica (grey short-tailed opossum). Journal of Anatomy 181 481-498.

Fleming W \& Saacke R 1972 Fine structure of the bovine oocyte from the mature graafian follicle. Journal of Reproduction and Fertility 29 203-213.

Frank K, Lyda R \& Kirkpatrick J 2005 Immunocontraception of captive exotic species. IV. Species differences in response to the porcine zona pellucida vaccine, timing of booster inoculations, and procedural failures. Zoo Biology 24 349-358.

Frankenberg S \& Selwood L 1998 An ultrastructural study of the role of an extracellular matrix during normal cleavage in a marsupial, the brushtail possum. Molecular Reproduction and Development 50 420-433.

Frankenberg S \& Selwood L 2001 Ultrastructure of oogenesis in the brushtail possum. Molecular Reproduction and Development 58 297-306.

Frankenberg S, Newell G \& Selwood L 1996 A light microscopic study of oogenesis in the brushtail possum Trichosurus vulpecula. Reproduction, Fertility, and Development 8 541-546.

Hardy C \& Braid A 2007 Vaccines for immunological control of fertility in animals. Revue Scientifique et Technique 26 461-470.

Henskens Y, Veerman E \& Nieuw Amerongen A 1996 Cystatins in health and disease. Biological Chemistry Hoppe-Seyler 377 71-86.

Homa S, Racowsky C \& McGaughey R 1986 Lipid analysis of immature pig oocytes. Journal of Reproduction and Fertility 77 425-434.

Kikuchi K, Ekwall H, Tienthai P, Kawai Y, Noguchi J, Kaneko H \& Rodriguez-Martinez H 2002 Morphological features of lipid droplet transition during porcine oocyte fertilisation and early embryonic development to blastocyst in vivo and in vitro. Zygote $10355-366$.

Kitchener A, Edds L, Molinia F \& Kay D 2002 Porcine zonae pellucidae immunization of tammar wallabies (Macropus eugenii): fertility and immune responses. Reproduction, Fertility, and Development 14 215-223.

Kos J, Sekirnik A, Kopitar G, Cimerman N, Kayser K, Stremmer A, Fiehn W \& Werle B 2001 Cathepsin S in tumours, regional lymph nodes and sera of patients with lung cancer: relation to prognosis. British Journal of Cancer 85 1193-1200.

Kress A 1996 A comparison of oocyte organelles in Monodelphis domestica with those of other marsupials and eutherians. Reproduction, Fertility, and Development 8 521-533.

Kress A \& Selwood L 2003 Conceptus polarity and cell-zona adhesion during early cleavage (fertilized tubal egg to 8-cell stage) in the marsupial Sminthopsis macroura. Cells, Tissues, Organs 175 9-24.

Kress A \& Selwood L 2004 Precedence of cell-zona adhesion over cell-cell adhesion during marsupial blastocyst formation prohibits morula formation and ensures that both the pluriblast and trophoblast are superficial. Cells, Tissues, Organs 177 87-103.

Lenarcic B, Gabrijelcic D, Rozman B, Drobnic-Kosorok M \& Turk V 1988 Human cathepsin B and cysteine proteinase inhibitors (CPIs) in inflammatory and metabolic joint diseases. Biological Chemistry Hoppe-Seyler 369 257-261.

Lyne A \& Hollis D 1983 Observations on Graafian follicles and their oocytes during lactation and after the removal of pouch young in the marsupials Isoodon macrourus and Perameles nasuta. American Journal of Anatomy $16641-61$.

Mahi-Brown C, Yanagimachi R, Nelson M, Yanagimachi H \& Palumbo N 1988 Ovarian histopathology of bitches immunized with porcine zonae pellucidae. American Journal of Reproductive Immunology and Microbiology 18 94-103.

Mehta P \& Ghadially F 1973 Articular cartilage in corn oil-induced lipoarthrosis. Annals of the Rheumatic Diseases 32 75-82. 
Merry N, Johnson M, Gehring C \& Selwood L 1995 Cytoskeletal organization in the oocyte, zygote, and early cleaving embryo of the stripe-faced dunnart (Sminthopsis macroura). Molecular Reproduction and Development 41 212-224.

Naz R, Gupta S, Gupta J, Vyas H \& Talwar A 2005 Recent advances in contraceptive vaccine development: a mini-review. Human Reproduction 20 3271-3283.

Pilton P \& Sharman G 1962 Reproduction in the marsupial Trichosurus vulpecula. Journal of Endocrinology 25 119-136.

Sathananthan H, Selwood L, Douglas I \& Nanayakkara K 1997 Early cleavage to formation of the unilaminar blastocyst in the marsupial Antechinus stuartii: ultrastructure. Reproduction, Fertility, and Development 9 201-212.

Selwood L 1992 Mechanisms underlying the development of pattern in marsupial embryos. Current Topics in Developmental Biology 27 $175-233$.

Selwood L 1994 Development of early cell lineages in marsupial embryos: an overview. Reproduction, Fertility, and Development 6 507-527.

Selwood L 2001 Mechanisms for pattern formation leading to axis formation and lineage allocation in mammals: a marsupial perspective. Reproduction 121 677-683.

Selwood L \& Smith D 1990 Time-lapse analysis and normal stages of development of cleavage and blastocyst formation in the marsupials the brown antechinus and the stripe-faced dunnart. Molecular Reproduction and Development 26 53-62.

Selwood L, Cui S \& Frankenberg S 1999 Oocyte and embryonic targets for immunocontraception in the brushtail possum: identification and isolation of genes and proteins. In Advances in the Biological Control of Possums, pp 54-59. Ed. G Sutherland. Wellington, New Zealand: The Royal Society of New Zealand.
Shorey C \& Hughes R 1973 Cyclical changes in the uterine endometrium and peripheral plasma concentrations of progesterone in the marsupial Trichosurus vulpecula. Australian Journal of Zoology 21 1-19.

Skinner S, Mills T, Kirchick H \& Dunbar B 1984 Immunization with zona pellucida proteins results in abnormal ovarian follicular differentiation and inhibition of gonadotropin-induced steroid secretion. Endocrinology 115 2418-2432.

Stoops M, Liu I, Shideler S, Lasley B, Fayrer-Hosken R, Benirschke K, Murata K, van Leeuwen E \& Anderson G 2005 Effect of porcine zonae pellucidae immunisation on ovarian follicular development and endocrine function in domestic ewes (Ovis aries). Reproduction, Fertility, and Development 18 667-676.

Taggart D, O'Brien H \& Moore H 1993 Ultrastructural characteristics of in vivo and in vitro fertilization in the grey short-tailed opossum, Monodelphis domestica. Anatomical Record 237 21-37.

Tesoriero J 1981 Early ultrastructural changes of developing oocytes in the dog. Journal of Morphology 168 171-179.

Turk V \& Bode W 1991 The cystatins: protein inhibitors of cysteine proteinases. FEBS Letters 285 213-219.

Ullmann S 1981 Observations on the primordial germ cells of bandicoots (Peramelidae, Marsupialia). Journal of Anatomy 132 581-595.

Ullmann S \& Butcher L 1996 Mammalian oocyte organelles with special reference to pleomorphic mitochondria and vacuole formation in marsupials. Reproduction, Fertility, and Development 8 491-508.

Received 9 April 2008

First decision 14 May 2008

Accepted 18 August 2008 